\title{
Microbial Air Sampling Plans in Cleanrooms According to Regulatory Authorities
}

\author{
Roberto Ligugnana* \\ Department of Applied Microbiology Laboratory Villa Cella, Italy \\ *Corresponding author: Roberto Ligugnana, Applied Microbiology Laboratory Villa Cella, Milan, Italy
}

\section{ARTICLE INFO}

Received: 㓞 November 22, 2019

Published: 櫘December 06, 2019

Citation: Roberto Ligugnana. Microbial Air Sampling Plans in Cleanrooms According to Regulatory Authorities. Biomed J Sci \& Tech Res 23(4)-2019. BJSTR. MS.ID.003937.

\begin{abstract}
The reported specific "Sampling Plan" has been prepared according to the requests of the Annex 1-Manufacture of Sterile Medicinal Products - Environmental Monitoring - Viable Monitoring. The Chapter 9.27 says that: "Continuous monitoring in grade A and $B$ areas should be undertaken for the full duration of critical processing, including equipment (aseptic set up) assembly and filling operations (i.e.: an understanding of functions and interactions of each clean area). The monitoring should be performed in such a way that all interventions, transient events and any system deterioration would be captured, and any risk caused by interventions of the monitoring operations is avoided". The document reports some examples of applications of the continuous monitoring of Cleanroom for viable microorganisms.
\end{abstract}

Keywords: Air Sampler; Aspirating Chamber; Aspirating Head; At Rest; Cleanroom; Cfu, Contamination; Bacteria; Culture Plate; Delay; Disinfection; End Of Shift Activity; Fraction Time; Fraction Number; Incubation; In Operation; Microorganisms; Petri Dish; Run, Standard; Sterilization; Unidirectional Laminar Flow

\section{Introduction}

USP guidelines for Cleanrooms state that the contamination monitoring is performed during the normal working day activity of each shift. The volume of 1000 litres of air should be collected at each sampling point. Air samples should be collected "at rest", "in operation", and "after process".

\section{Sampling Plan}

The sampling plan, part of a SOP (Standard Operating Procedures), should be written prior to begin an ongoing environmental program. A robustus air sampling program is and developed based upon the size of the area, number of hoods and after a risk assessment. In order to trend the number and type of microorganisms on a day / weekly / monthly basis, it is necessary to sample with the same method / protocol each time. Areas to be sampled are indicated on a floor plan of the Cleanroom and should include laminar flow benches, BSC, Isolator, RABS, buffer zone, gowning areas, and anteroom areas.

\section{L Of Air to Be Collected}

The operators, employing a process involving a single head active air sampler, will need to perform several manipulations

regarding preparation and culture plate transfer. Working time and the risk of contamination can be reduced with the use of a two or three aspirating head format sampler. The sampler can be prepared and programmed prior to starting daily activity and the culture plates collected at the end of the shift. "Shift working time" differs from company to company, and with cyclical processing circumstances, from time to time. In this document, we report several examples using TRIO.BAS DUO and TRIO.BAS TRIO to demonstrate sampling flexibility. In addition to the programming samples provided, note instrument start times may also be programmed with the "delay" function (Figures 1 \& 2).

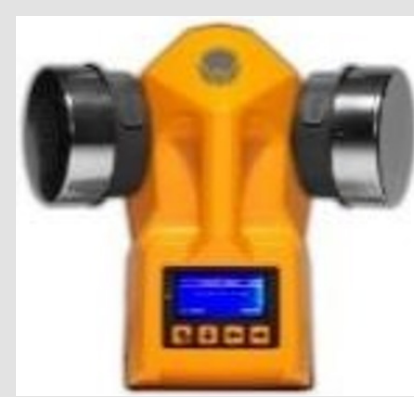

Figure 1. 


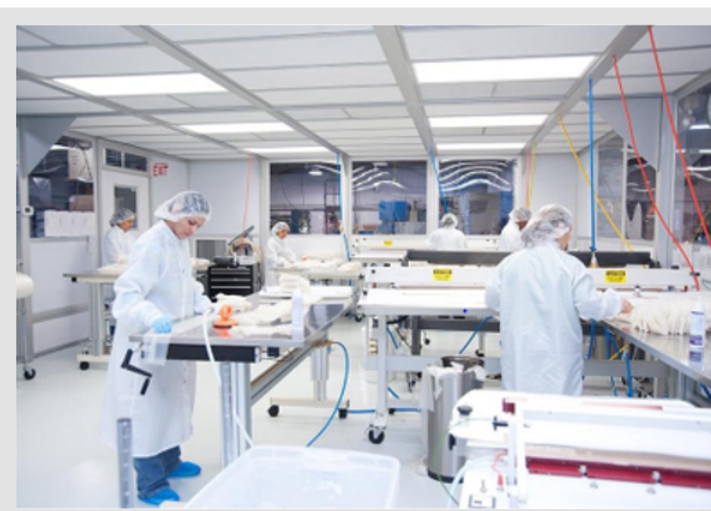

Figure 2.

\section{Materials}

Microbial air sampler with 2 aspirating heads, $100 \mathrm{~mm}$ Petri dish with TSA agar culture media, "Daily Shift" sterile certified aspirating heads.

\section{Protocol}

Hours long sampling spans can be programmed by different "delay", "fraction time", and "fraction number". Are here reported several examples: 1.000 litres of air samples during different working shift runs. The cycles are stored in the TRIO.BAS air sampler memory and then can be transferred to a P.C., via one of the data transfer accessory options (Tables $1 \& 2$ ).

Table 1.

EXAMPLE OF 2h.20min WORK SHIFT

SAMPLER CFG: VOL - 1000 LTS - OK - DELAY - 0 -OK - FRACTION NUM. - 2 -OK - FR. TIME - 60 - OK - HEAD MODE SQS - OK - GO - HEAD $1 \& 2$ - OK Duo $100 \mathrm{l} / \mathrm{m}$ prova 2 fraction $-1000 \mathrm{l} / \mathrm{m}$

\begin{tabular}{|c|c|c|c|c|c|c|}
\hline Head & Fraction & Starting Hour & Time Test & Sampler \% & $\mathrm{L} / \mathrm{m}$ session & End of test \\
\hline 1 & A & 9 & $5,00 \mathrm{MIN}$ & $50 \%$ & 500 & 9.05 \\
\hline 1 & B & 10.05 & $5,00 \mathrm{MIN}$ & $100 \%$ & 500 & 10.10 \\
\hline 2 & A & 10.10 & 5,00 MIN & $50 \%$ & 500 & 10.15 \\
\hline 2 & B & 11.15 & 5,00 MIN & $100 \%$ & 500 & 11.20 \\
\hline
\end{tabular}

Table 2.

EXAMPLE OF 3h.21min WORK SHIFT

SAMPLER CFG: VOL - 1000 LTS - OK - DELAY - 0 - OK - FRACTION NUM. - 3 -OK - FR. TIME - 45 - OK - HEAD MODE SQS - OK - GO - HEAD 1 \&2 - OK Duo $100 \mathrm{l} / \mathrm{m}$ prova 3 fraction $-1000 \mathrm{l} / \mathrm{m}$

\begin{tabular}{|c|c|c|c|c|c|c|}
\hline Head & Fraction & Starting Hour & Time Test & Sampler \% & $\mathrm{L} / \mathrm{m}$ session & End of test \\
\hline 1 & A & 9.00 & 3,30 MIN & $33 \%$ & 333 & 9.03 \\
\hline 1 & B & 9.48 & 3,30 MIN & $66 \%$ & 333 & 9.52 \\
\hline 1 & $\mathrm{C}$ & 10.37 & 3,30 MIN & $100 \%$ & 333 & 10.40 \\
\hline 2 & A & 10.40 & 3,30 MIN & $33 \%$ & 333 & 10.44 \\
\hline 2 & $\mathrm{~B}$ & 11.29 & 3,30 MIN & $66 \%$ & 333 & 11.32 \\
\hline 2 & $\mathrm{C}$ & 12.17 & 3,30 MIN & $100 \%$ & 333 & 12.21 \\
\hline
\end{tabular}

\section{Standard Operating Procedure (Sop)}

The SOP-Standard Operating Procedures for environmental monitoring can be downloaded or requested from ORUM International S.r.l.

\section{Practical Application}

Starting "At rest" at 09:00 / "In operation" at 10:05 / "At end" at 11:20a.m.

Starting "At rest" at 09:00a.m. "In operation" at 10:37 / "At end" at 12:21p.m.

The continuous monitoring of the viable microorganisms can be expanded up to 6 or 8 hours with the appropriate use of the "delay", "fraction number" and "fraction time" functions of the microbial air samplers.

\section{Conclusion}

This protocol conforms what the Regulatory Authorities request: "9.27 Continuous monitoring in grade A an B areas should be undertaken for the full duration of critical processing, including equipment, assembling and filling operation".

\section{References}

1. Annex 1, Manufacture of Sterile Medicinal Products - Environmental Monitoring - Viable Monitoring. 
ISSN: 2574-1241

DOI: 10.26717/BJSTR.2019.23.003937

Roberto Ligugnana. Biomed J Sci \& Tech Res

(C) This work is licensed under Creative

Submission Link: https://biomedres.us/submit-manuscript.php

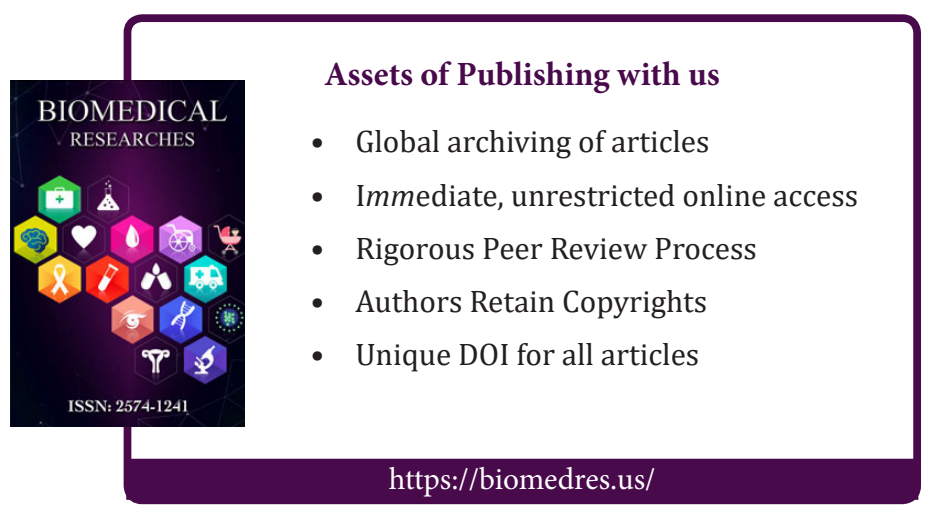

